\title{
What You See Is What You Get.Get: Surface Transparency and Ambiguity of Nominalizing Reduplication in American Sign Language
}

\section{Supplementary Video File Information}

Video 1 (VOTE, VOTE.NMLZ-RED): A signer produces the verb VOTE (vote) and its nominalized counterpart VOTE.NMLZ-RED (manual form for election or ballot), each in a carrier sentence: IX SAY _ YESTERDAY (She/he said _ yesterday). The sign SAY is produced with the index finger tapping the chin and the sign YESTERDAY is produced with the thumb touching near the chin and then near the ear. The target sign is the sign produced between these two instances of facial contact. The first portion of the video is IX 3 P-SG SAY VOTE YESTERDAY (She/he said vote yesterday) and the second is IX $3 \mathrm{P}-\mathrm{SG}$ SAY VOTE.NMLZ-RED YESTERDAY (She/he said election/ballot yesterday). The signer in this video may be disambiguating by co-producing the nominalized sign with a mouthing corresponding to election. This video corresponds to the image stills presented in Figure 3 and Figure 4.

Video 2 (FLY, FLY.NMLZ-RED): A signer produces the verb FLY ( $f l y)$ and its nominalized counterpart FLY.NMLZ-RED (manual form for airplane or flight), each in a carrier sentence: IX SAY __ YESTERDAY (She/he said__ yesterday). The sign SAY is produced with the index finger tapping the chin and the sign YESTERDAY is produced with the thumb touching near the chin and then near the ear. The target sign is the sign produced between these two instances of facial contact. The first portion of the video is IX 3 P-SG SAY FLY YESTERDAY (She/he said fly yesterday) and the second is IX $3 \mathrm{P}-\mathrm{SG}$ SAY FLY.NMLZ-RED YESTERDAY (She/he said airplane/flight yesterday). The signer in this video may be disambiguating by co-producing the nominalized sign with a mouthing corresponding to plane. 
Video 3 (ACCEPT, ACCEPT.NMLZ-RED): A signer produces the verb ACCEPT (accept) and its nominalized counterpart ACCEPT.NMLZRED (acceptance), each in a carrier sentence: IX SAY __ YESTERDAY (She/he said__ yesterday'). The sign SAY is produced with the index finger tapping the chin and the sign YESTERDAY is produced with the thumb touching near the chin and then near the ear. The target sign is the sign produced between these two instances of facial contact. The first portion of the video is IX 3 P-SG SAY ACCEPT YESTERDAY (She/he said accept yesterday) and the second is $\mathrm{IX}_{3 \mathrm{P}-\mathrm{SG}}$ SAY ACCEPT.NMLZ-RED YESTERDAY (She/he said acceptance yesterday). 


\section{Article Figures}
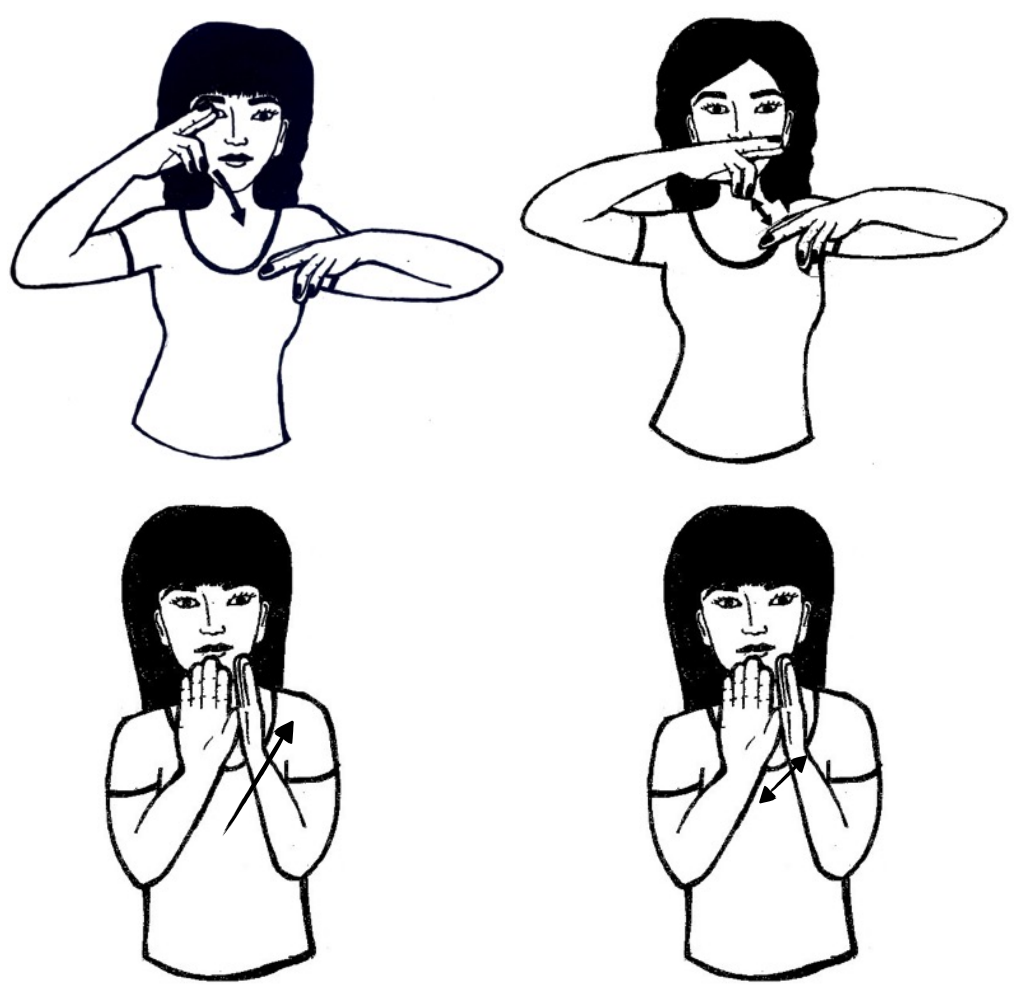

Figure 1: Illustration of the verbal and derived nominal forms in (1). The verbal forms SIT 'sit' (top left) and DEVELOP 'develop' (bottom left) both contain a single, elongated movement, whereas the derived nominal forms SIT.NMLZ-RED 'chair' (top right) and DEVELOP.NMLZ-RED 'development' (bottom right) are produced with short, tense, repeated movement. 


\section{Event Process}

(Path Movement)

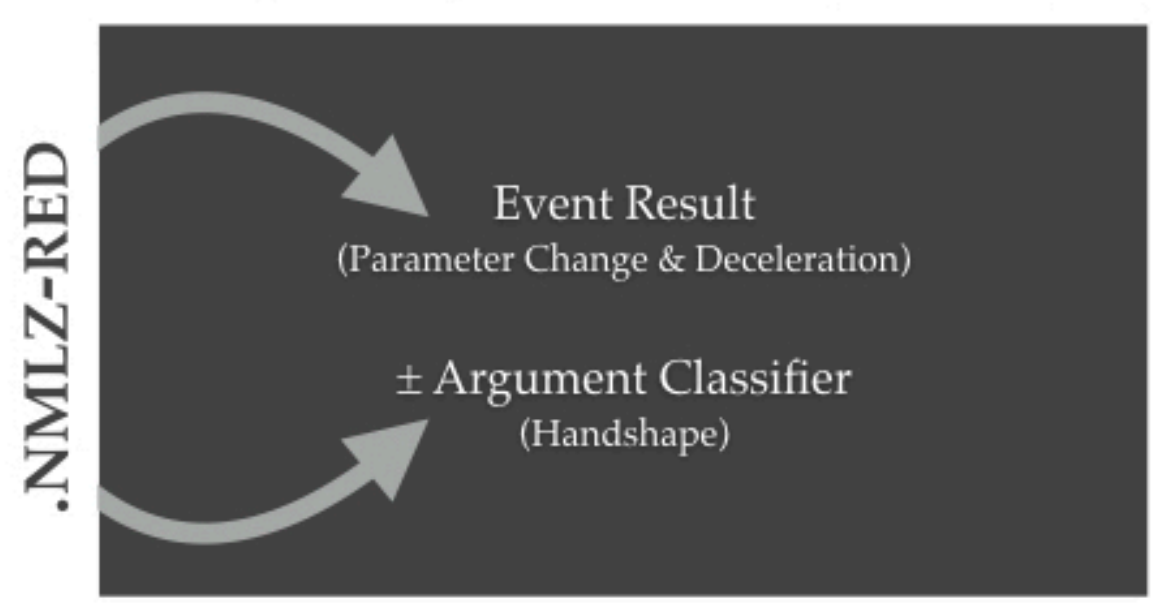

Figure 2: Structural schematic of the nominalizing reduplication process in ASL. The lower verbal layer contains information pertaining to the event result and may also contain an argument-introducing classifier. This is the layer targeted for nominalization by .NMLZ-RED, which may anchor its meaning to either the event result or the argument classifier. 


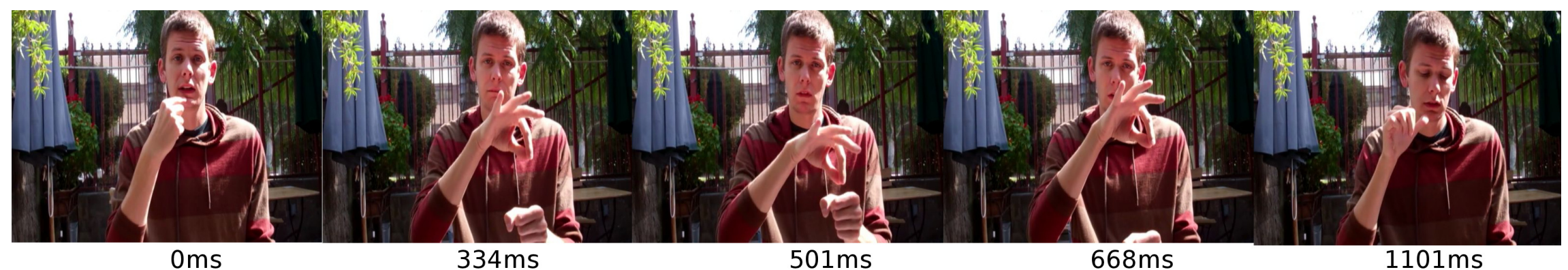

Figure 3: Time-stamped video stills of the sign vOTE 'vote'. The single contact movement of this verbal production occurs just subsequent to the middle $(501 \mathrm{~ms})$ frame. 


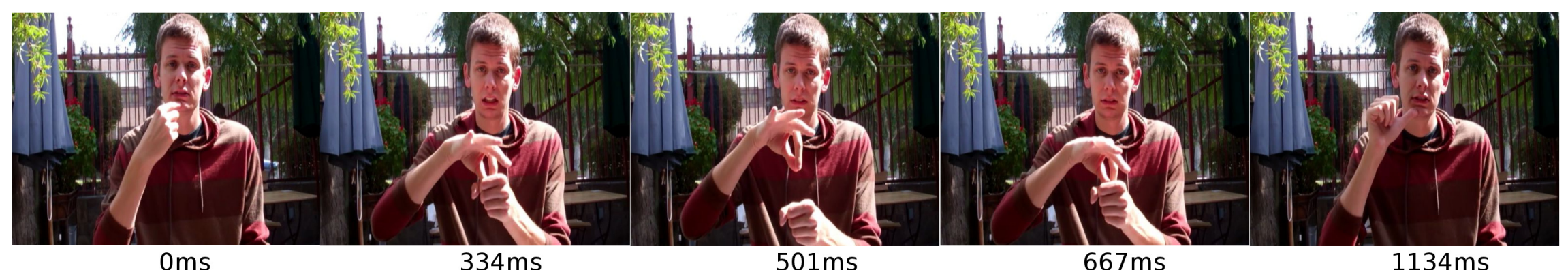

Figure 4: Time-stamped video stills of the sign VOTE.NMLZ-RED 'ballot'/'election'. The two instances of contact movement occur in the second (334ms) and fourth $(667 \mathrm{~ms})$ frames. 


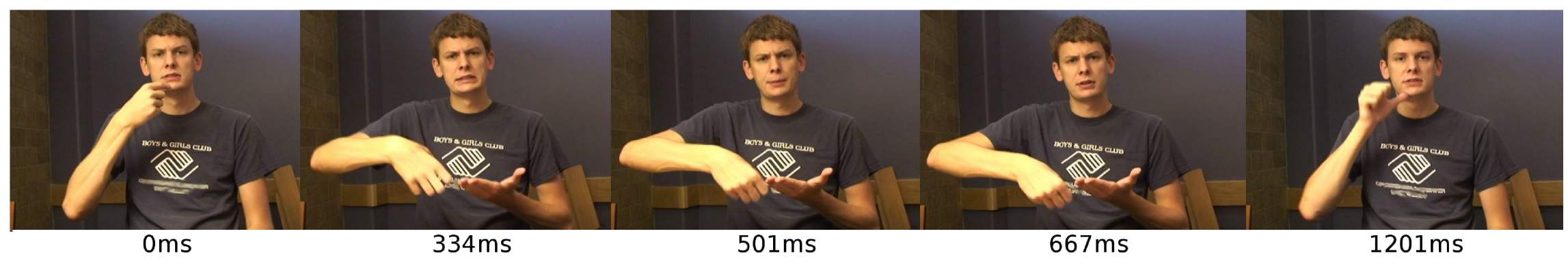

Figure 5: Time-stamped video stills of the reduplicated aperture change nominal STAPLE-WITH-STAPLER.NMLZ-RED 'stapler'. 


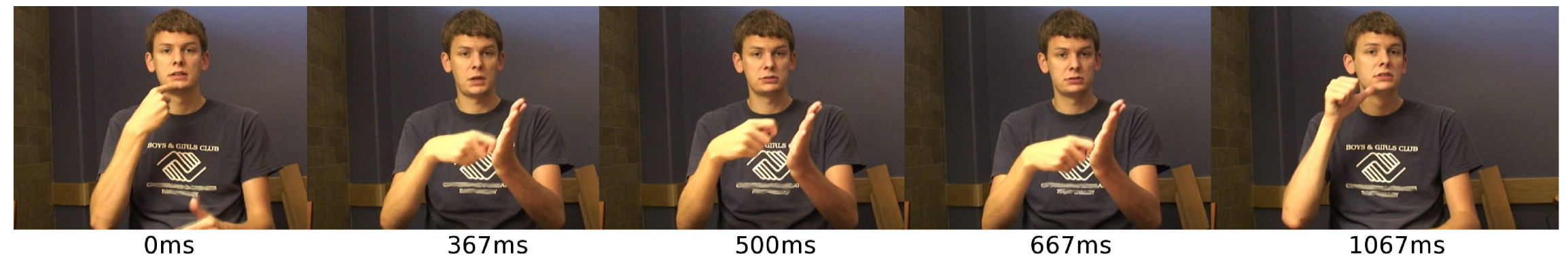

Figure 6: Time-stamped video stills of the reduplicated orientation change nominal STRIKE-MATCH.NMLZ-RED 'match'. 


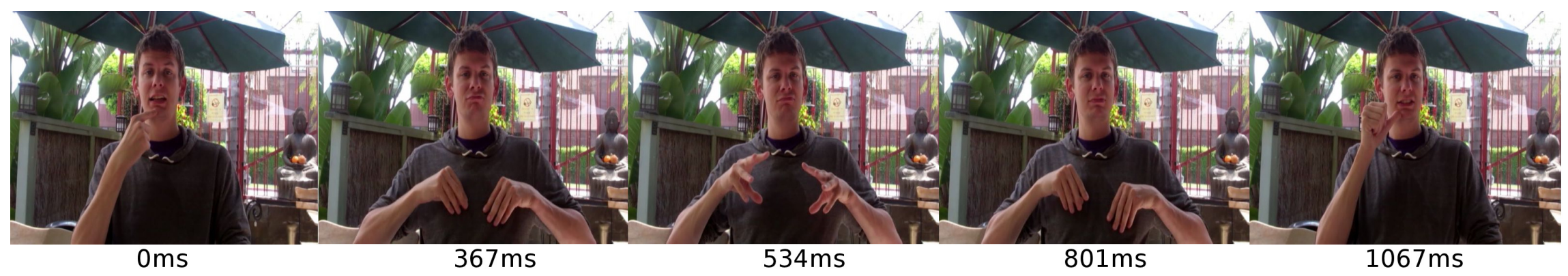

Figure 7: Time-stamped video stills of the reduplicated aperture change nominal ACCEPT.NMZ-RED 'acceptance'. 